\title{
Cognitive Intervention As an Early Non-pharmacological Strategy in Alzheimer's Disease: A Translational Perspective
}

\author{
Sarah W. Gehres ${ }^{1 \dagger}$, Andreia Rocha ${ }^{1 \dagger}$, Antoine Leuzy ${ }^{2}$, Cássio M. Loss ${ }^{1}$, \\ Giordano G. Viola ${ }^{3}$ and Eduardo R. Zimmer ${ }^{1,4 *}$ \\ ${ }^{1}$ Department of Biochemistry, Federal University of Rio Grande do Sul, Porto Alegre, Brazil, ${ }^{2}$ Department NVS, Center for \\ Alzheimer Research, Division of Translational Alzheimer Neurobiology, Karolinska Institutet, Stockholm, Sweden, ${ }^{3}$ Department \\ of Physiology, Federal University of Sergipe, São Cristóvão, Brazil, ${ }^{4}$ Brain Institute of Rio Grande do Sul (Bralns), Pontifical \\ Catholic University of Rio Grande do Sul, Porto Alegre, Brazil
}

Keywords: Alzheimer's disease, cognitive intervention, cognitive reserve, dementia, environmental enrichment

\section{OPEN ACCESS}

Edited by:

Lucas Sedeño,

Institute of Cognitive Neurology

(INECO), Argentina

Reviewed by:

James C. Vickers,

University of Tasmania, Australia

*Correspondence:

Eduardo R. Zimmer

eduardo.zimmer@ufrgs.br

${ }^{\dagger}$ These authors have contributed equally to this work.

Received: 09 September 2016 Accepted: 08 November 2016 Published: 29 November 2016

Citation:

Gehres SW, Rocha A, Leuzy A, Loss CM, Viola GG and Zimmer ER (2016) Cognitive Intervention As an Early Non-pharmacological Strategy in Alzheimer's Disease: A Translational Perspective.

Front. Aging Neurosci. 8:280. doi: 10.3389/fnagi.2016.00280
Brain amyloid- $\beta(\mathrm{A} \beta)$ accumulation is currently considered the main causative pathophysiological event in Alzheimer's disease (AD) (Hardy and Higgins, 1992; Karran et al., 2011). Importantly, this process is thought to precede the onset of $\mathrm{AD}$ clinical symptoms by more than two decades, indicating that early therapeutic strategies prior to symptomatology offer the best chance of success. In line with this, there is growing attention being paid to the concept of cognitive reserve (CR) (Stern et al., 1994; Stern, 2002). CR concept is based on extensive epidemiological data indicating that those with higher lifetime levels of social, physical, and cognitive engagement have a lower risk of developing dementia despite the presence of brain pathology (Fratiglioni et al., 2004; Nithianantharajah and Hannan, 2009). Recently, cognitive intervention (CI)-such as cognitive training (Bahar-Fuchs et al., 2013), cognitive stimulation (Woods et al., 2012), and cognitive rehabilitation (Clare et al., 2003)-has emerged as a potential non-pharmacological strategy for the treatment and prevention of AD (Gates and Sachdev, 2014). Although based upon distinct theoretical constructs, these CI strategies are frequently not distinguished in clinical trials.

Clinical data are supported by compelling evidence from experimental studies, which have demonstrated that early-life exposure to environmental enrichment (EE), an experimental method of $\mathrm{CR}$ in animals, is able to prevent memory decline in AD-like animal models (Valero et al., 2011; Verret et al., 2013; Polito et al., 2014). However, the precise mechanisms behind this phenomenon remain elusive. Environmental enrichment for animals involves stimulating not only their senses (e.g., smell, sight, touch), but also stimulating their ability to learn and adapt when exposed to novelty and challenge, including via stimulation of innate behaviors, such as foraging and partner seeking. The same line of thinking can be applied to humans, on its own scale. This would translate into exercise, social interaction, learning new things, exploring new environments, keeping the brain active with cognitive training, i.e., challenging the brain to self-adapt to novelty.

In a recent study, published in Frontiers in Aging Neuroscience, Bezzina et al. (2015) reared mice harboring a human pathological double mutation in the amyloid precursor protein gene (APP, model tg2576) in standard or enriched housing conditions for 10 weeks, starting at 3 months of age (pre-A $\beta$ plaque phase). Two weeks after EE exposure, transgenic animals housed in standard or EE cages presented similar seizure susceptibility to pentylenetetrazole (PTZ), a wellestablished GABA receptor antagonist. Moreover, the frequency of interictal spikes-indexed by electroencephalography (EEG) - after EE was similar between groups. In short, the authors show that $\mathrm{EE}$ is not capable of halting the $\mathrm{A} \beta$-induced aberrant neuronal activity in the Tg2576 AD-like model. These findings indicate that preventive effects of EE on cognitive decline are not necessarily 
related to changes in neuronal activity. Although negative, these findings are very relevant since they help rule out abnormal neuronal activity as a mechanism by which EE exerts its beneficial impact on memory performance.

By contrast, data analyzing the effects of EE on neurogenesis in AD-like animal models have been mostly positive. EE exposure for 6 months was able to restore the impaired hippocampal neurogenesis of a triple transgenic $\mathrm{AD}$-like mouse model (3xTg-AD, a model harboring human mutations in: APP (Swedish, KM670/671NL), Tau (MAPT P301L) and Presenilin 1 (PSEN1 M146V) (Rodríguez et al., 2008, 2011). The density analysis of hippocampal proliferating cells was performed by immunohistochemistry targeting the presence of phosphorylated Histone $\mathrm{H} 3$ (HH3), and their potential neuronal and glial phenotype by co-localizing the proliferating cells with the immature neuronal marker doublecortin (DCX), the mature neuronal marker $(\mathrm{NeuN})$ or the specific astroglial marker (GFAP). This very interesting study showed a significant increase in neuronal proliferating cells (HH3/DCX or $\mathrm{HH} 3 / \mathrm{NeuN}$ positive) in the dentate gyrus of transgenic animals housed in EE. These results, together with the findings of other experimental studies, suggest the stimulation of adult neurogenesis as a potential process by which EE prevents cognitive impairment in AD-like models (Wolf et al., 2006; Herring et al., 2009; Mirochnic et al., 2009; Lahiani-Cohen et al., 2011). In this context, recent evidence indicates that the benefitial effects of $\mathrm{EE}$ are more related to the reorganization of the neuronal network built by these newborn neurons than to the absolute number of newborn neurons per se (Vivar et al., 2016).

However, the impact of $\mathrm{EE}$ on $\mathrm{A} \beta$ levels in the brain is still largely inconclusive (Mainardi et al., 2014; Polito et al., 2014; Rodríguez et al., 2015). Male APP23, a mouse model expressing the human APP Swedish double mutation, exposed to EE starting at 3 months of age until 7 or 18 months showed a marked cognitive improvement without altering soluble $A \beta$ (7 monthold) but reducing the number of $A \beta$ plaques at 18 month-old. These data indicate that EE effect seems to affect $A \beta$ in a distinct manner.

We are of the opinion that deciphering the underlying mechanisms of EE can provide important information about novel therapeutic targets. With currently available treatments for $\mathrm{AD}$ being palliative at best and ineffective at worstresults from drug trials targeting brain $\mathrm{A} \beta$ have been either inconclusive or negative-non-pharmacological interventions stimulating cognition in humans hold a measure of promise, not only for delaying symptomatic onset and the loss of cognitive functions, but also in terms of unveiling potential targets.

The take-home message from EE studies is clear, however, and translates to human disease: interventions must occur early on in the disease course, prior to substantial cognitive deficits (Figure 1). The question then becomes: how to translate these findings to clinical research? A promising approach is to study individuals in the so-called "preclinical" or "asymptomatic atrisk" stage of $\mathrm{AD}$ [please see revised definition of preclinical AD in Sperling et al. (2011) and Dubois et al. (2016)].

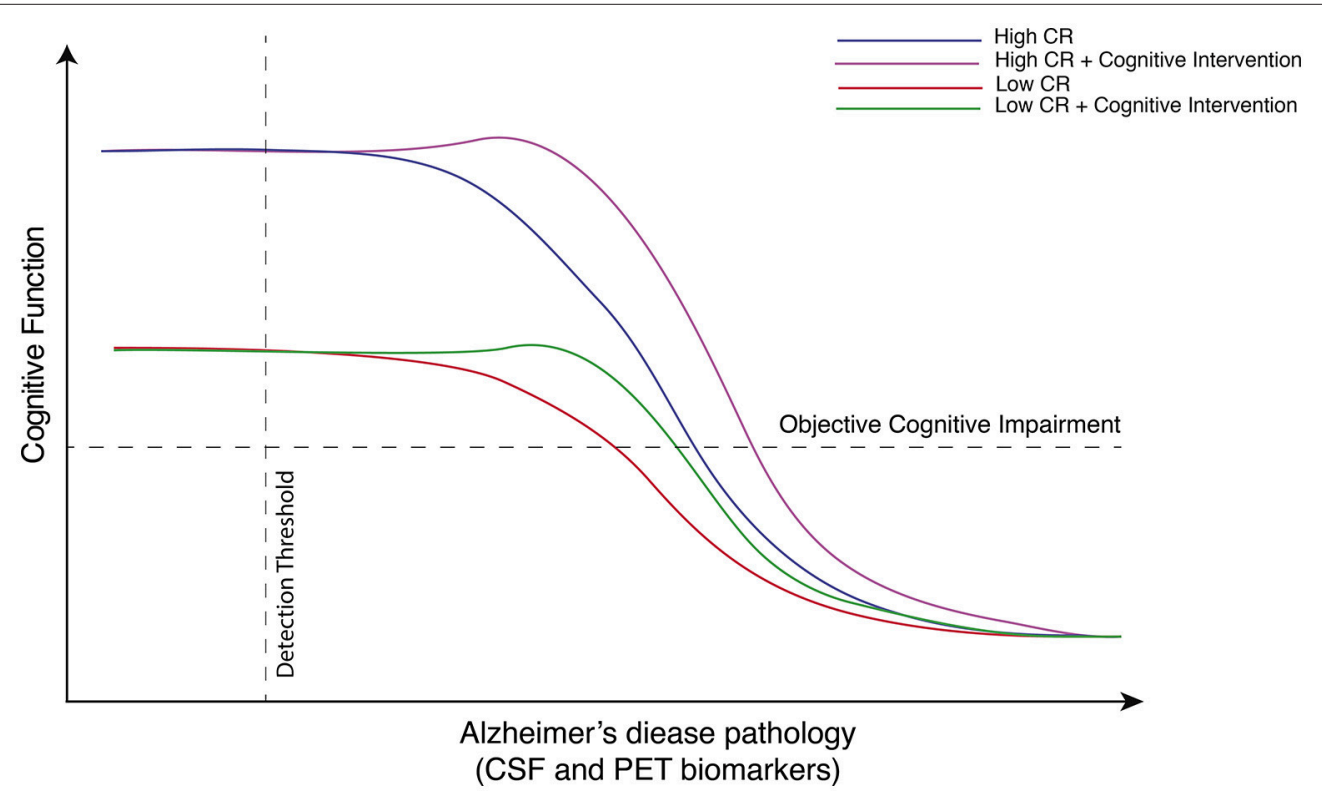

FIGURE 1 | Hypothetical model depicting the beneficial effects of cognitive intervention on cognitive function on low and high cognitive reserve individuals as a function of Alzheimer's disease neuropathology. This hypothetical model exemplifies changes in cognitive function changes over Alzheimer's disease (AD) continuum in individuals with high and low cognitive reserve (CR). When AD pathology is absent or possibly below detectable levels using current biomarkers, individuals with high CR have been shown to perform better on neurophysiological tests than individual with low CR. While cognitive intervention probably cannot ultimately arrest the progression of AD pathology, as measured by imaging or fluid based biomarkers, it can potentially delay the onset and progression of cognitive symptoms. In keeping with the observation that individuals with high CR can tolerate greater levels of pathology prior to declines in cognitive performance, the rate of decline is greater in the high CR group. 
Defined by the absence of cognitive deficits despite in situ $\mathrm{AD}$ pathology_established using cerebrospinal fluid (CSF) or positron emission tomography (PET) based biomarkers-crosssectional and longitudinal studies suggest this preclinical phase to span some 20-30 years (Jansen et al., 2015). Based on this, non-pharmacological interventions directed at CR could be encouraged in so called "amyloid-positive" individuals believed to be on the path to symptomatic AD. In this way, we refer to the concepts of primary and secondary prevention, as defined by Gates et al. (2011). Primary prevention in the form of early intervention, aimed at preclinical individuals, in order to keep them asymptomatic for as long as possible; and secondary prevention in the form of cognitive training and stimulation for those who already show subtle and early cognitive impairment [such as those in the mild cognitive impairment (MCI) phase], to slow the cognitive decline into AD dementia.

While follow-up studies with longer assessment periods are essential for more definitive data, early results from several multidomain non-pharmacologic intervention studies-addressing diet, exercise, cognitive training and vascular risk monitoringsuggest that cognitive functioning can be maintained or even improved in at-risk elderly individuals (Ngandu et al., 2015). The incorporation of amyloid biomarkers into such intervention trials could stand as a valuable adjunct to cognitive and functional outcome measures, possibly allowing for more rapid translational estimations.

Though EE stimulation has been shown to be a promising approach for delaying disease progression in animal models mimicking early onset autosomal dominant $\mathrm{AD}$, which accounts for less than $5 \%$ of cases, what about the far more common age-related (sporadic) and multifactorial form of AD? How does $\mathrm{EE}$ impact on sporadic models of $\mathrm{AD}$ ? Here stands a problem in that models replicating sporadic $\mathrm{AD}$ are currently lacking. In our opinion, major developments in modeling the

\section{REFERENCES}

Bahar-Fuchs, A., Clare, L., and Woods, B. (2013). Cognitive training and cognitive rehabilitation for persons with mild to moderate dementia of the Alzheimer's or vascular type: a review. Alzheimers Res. Ther. 5, 35. doi: 10.1186/alzrt189

Bezzina, C., Verret, L., Halley, H., Dahan, L., and Rampon, C. (2015). Environmental enrichment does not influence hypersynchronous network activity in the Tg2576 mouse model of Alzheimer's disease. Front. Aging Neurosci. 7:178. doi: 10.3389/fnagi.2015.00178

Clare, L., Woods, R. T., Moniz Cook, E. D., Orrell, M., and Spector, A. (2003). Cognitive rehabilitation and cognitive training for early-stage Alzheimer's disease and vascular dementia. Cochrane Database Syst. Rev. CD003260. doi: 10.1002/14651858.CD003260. [Epub ahead of print].

Dubois, B., Hampel, H., Feldman, H. H., Scheltens, P., Aisen, P., Andrieu, S., et al. (2016). Preclinical Alzheimer's disease: definition, natural history, and diagnostic criteria. Alzheimers. Dement. 12, 292-323. doi: 10.1016/j.jalz.2016.02.002

Fratiglioni, L., Paillard-Borg, S., and Winblad, B. (2004). An active and socially integrated lifestyle in late life might protect against dementia. Lancet Neurol. 3, 343-353. doi: 10.1016/S1474-4422(04)00767-7

Gates, N. J., and Sachdev, P. (2014). Is cognitive training an effective treatment for preclinical and early Alzheimer's disease? J. Alzheimers Dis. 42(Suppl. 4), S551-S559. doi: 10.3233/JAD-141302

Gates, N. J., Sachdev, P. S., Fiatarone Singh, M. A., and Valenzuela, M. (2011). Cognitive and memory training in adults at risk of dementia: sporadic form of $\mathrm{AD}$ in rodents will potentially come from the incorporation of genetic variations that increase the risk of developing $\mathrm{AD}$, such as the $\varepsilon 4$ allele of apolipoprotein $\mathrm{E}$ (APOE) gene, or the $\mathrm{R} 47 \mathrm{H}$ allele of the triggering receptor expressed on myeloid cells 2 (TREM2). Animal models expressing such combinations of genetic risk factors may better resemble sporadic $\mathrm{AD}$ and ultimately translate into more relevant disease models.

In conclusion, study designs aiming toward deciphering EE specific mechanisms are vital and can potentially drive nonpharmacological strategies. Here we make an argument that the combination of EE with models that better mimic the sporadic form of $\mathrm{AD}$, as well as CSF- and PET-based biomarkers, will potentially provide important insights into $\mathrm{AD}$ pathophysiology and highlight novel therapeutic targets, with strong translational value.

\section{AUTHOR CONTRIBUTIONS}

SG and AR were responsible for reviewing the literature and drafting the manuscript. $\mathrm{CL}, \mathrm{GV}$ and $\mathrm{AL}$ were responsible for revising the manuscript. EZ was responsible for the conceptualization and drafting the manuscript. All authors critically revised the final version of the manuscript.

\section{ACKNOWLEDGMENTS}

This work was supported by the Conselho Nacional de Desenvolvimento Científico e Tecnológico (CNPq, Brazil) [460594/2014-1], the INCT for Excitotoxicity and Neuroprotection [573677/2008-5], the Coordenação de Aperfeiçoamento de Pessoal de Nível Superior (CAPES, Brazil) [PNPD], and the Fundação de Amparo à Pesquisa do Estado do Rio Grande do Sul (FAPERGS). a systematic review. BMC Geriatr. 11:55. doi: 10.1186/1471-231 8-11-55

Hardy, J. A., and Higgins, G. A. (1992). Alzheimer's disease: the amyloid cascade hypothesis. Science 256, 184-185. doi: 10.1126/science. 1566067

Herring, A., Ambrée, O., Tomm, M., Habermann, H., Sachser, N., Paulus, W., et al. (2009). Environmental enrichment enhances cellular plasticity in transgenic mice with Alzheimer-like pathology. Exp. Neurol. 216, 184-192. doi: 10.1016/j.expneurol.2008.11.027

Jansen, W. J., Ossenkoppele, R., Knol, D. L., Tijms, B. M., Scheltens, P., Verhey, F. R., et al. (2015). Prevalence of cerebral amyloid pathology in persons without dementia: a meta-analysis. JAMA 313, 1924-1938. doi: 10.1001/jama.2015.4668

Karran, E., Mercken, M., and De Strooper, B. (2011). The amyloid cascade hypothesis for Alzheimer's disease: an appraisal for the development of therapeutics. Nat. Rev. Drug Discov. 10, 698-712. doi: 10.1038/nrd3505

Lahiani-Cohen, I., Lourbopoulos, A., Haber, E., Rozenstein-Tsalkovich, L., Abramsky, O., Grigoriadis, N., et al. (2011). Moderate environmental enrichment mitigates tauopathy in a neurofibrillary tangle mouse model. J. Neuropathol. Exp. Neurol. 70, 610-621. doi: 10.1097/NEN.0b013e3182 $21 \mathrm{bfab}$

Mainardi, M., Di Garbo, A., Caleo, M., Berardi, N., Sale, A., and Maffei, L. (2014). Environmental enrichment strengthens corticocortical interactions and reduces amyloid-beta oligomers in aged mice. Front. Aging Neurosci. 6:1. doi: 10.3389/fnagi.2014.00001

Mirochnic, S., Wolf, S., Staufenbiel, M., and Kempermann, G. (2009). Age effects on the regulation of adult hippocampal neurogenesis by physical activity and 
environmental enrichment in the APP23 mouse model of Alzheimer disease. Hippocampus 19, 1008-1018. doi: 10.1002/hipo.20560

Ngandu, T., Lehtisalo, J., Solomon, A., Levälahti, E., Ahtiluoto, S., Antikainen, R., et al. (2015). A 2 year multidomain intervention of diet, exercise, cognitive training, and vascular risk monitoring versus control to prevent cognitive decline in at-risk elderly people (FINGER): a randomised controlled trial. Lancet 385, 2255-2263. doi: 10.1016/S0140-6736(15)60461-5

Nithianantharajah, J., and Hannan, A. J. (2009). The neurobiology of brain and cognitive reserve: mental and physical activity as modulators of brain disorders. Prog. Neurobiol. 89, 369-382. doi: 10.1016/j.pneurobio.2009.10.001

Polito, L., Chierchia, A., Tunesi, M., Bouybayoune, I., Kehoe, P. G., Albani, D., et al. (2014). Environmental enrichment lessens cognitive decline in APP23 mice without affecting brain sirtuin expression. J. Alzheimers Dis. 42, 851-864. doi: $10.3233 /$ JAD-131430

Rodríguez, J. J., Jones, V. C., Tabuchi, M., Allan, S. M., Knight, E. M., Laferla, F. M., et al. (2008). Impaired adult neurogenesis in the dentate gyrus of a triple transgenic mouse model of Alzheimer's disease. PLoS ONE 3:e2935. doi: 10.1371/journal.pone.0002935

Rodríguez, J. J., Noristani, H. N., Olabarria, M., Fletcher, J., Somerville, T. D., Yeh, C. Y., et al. (2011). Voluntary running and environmental enrichment restores impaired hippocampal neurogenesis in a triple transgenic mouse model of Alzheimer's disease. Curr. Alzheimers Res. 8, 707-717. doi: 10.2174/156720511797633214

Rodríguez, J. J., Noristani, H. N., and Verkhratsky, A. (2015). Microglial response to Alzheimer's disease is differentially modulated by voluntary wheel running and enriched environments. Brain Struct. Funct. 220, 941-953. doi: 10.1007/s00429-013-0693-5

Sperling, R. A., Aisen, P. S., Beckett, L. A., Bennett, D. A., Craft, S., Fagan, A. M., et al. (2011). Toward defining the preclinical stages of Alzheimer's disease: recommendations from the National Institute on Aging-Alzheimer's Association workgroups on diagnostic guidelines for Alzheimer's disease. Alzheimers Dement. 7, 280-292. doi: 10.1016/j.jalz.2011.03.003

Stern, Y. (2002). What is cognitive reserve? Theory and research application of the reserve concept. J. Int. Neuropsychol. Soc. 8, 448-460. doi: $10.1017 /$ S1355617702813248
Stern, Y., Gurland, B., Tatemichi, T. K., Tang, M. X., Wilder, D., and Mayeux, R. (1994). Influence of education and occupation on the incidence of Alzheimer's disease. JAMA 271, 1004-1010. doi: 10.1001/jama.1994.035103700 56032

Valero, J., España, J., Parra-Damas, A., Martin, E., Rodríguez-Álvarez, J., and Saura, C. A. (2011). Short-term environmental enrichment rescues adult neurogenesis and memory deficits in $\mathrm{APP}(\mathrm{Sw}$,Ind) transgenic mice. PLoS ONE 6:e16832. doi: 10.1371/journal.pone.0016832

Verret, L., Krezymon, A., Halley, H., Trouche, S., Zerwas, M., Lazouret, M., et al. (2013). Transient enriched housing before amyloidosis onset sustains cognitive improvement in Tg2576 mice. Neurobiol. Aging 34, 211-225. doi: 10.1016/j.neurobiolaging.2012.05.013

Vivar, C., Peterson, B. D., and Van Praag, H. (2016). Running rewires the neuronal network of adult-born dentate granule cells. Neuroimage 131, 29-41. doi: 10.1016/j.neuroimage.2015.11.031

Wolf, S. A., Kronenberg, G., Lehmann, K., Blankenship, A., Overall, R., Staufenbiel, M., et al. (2006). Cognitive and physical activity differently modulate disease progression in the amyloid precursor protein (APP)-23 model of Alzheimer's disease. Biol. Psychiatry 60, 1314-1323. doi: 10.1016/j.biopsych.2006.04.004

Woods, B., Aguirre, E., Spector, A. E., and Orrell, M. (2012). Cognitive stimulation to improve cognitive functioning in people with dementia. Cochrane Database Syst. Rev. CD005562. doi: 10.1002/14651858.CD005562.pub2. [Epub ahead of print].

Conflict of Interest Statement: The authors declare that the research was conducted in the absence of any commercial or financial relationships that could be construed as a potential conflict of interest.

Copyright (C) 2016 Gehres, Rocha, Leuzy, Loss, Viola and Zimmer. This is an openaccess article distributed under the terms of the Creative Commons Attribution License (CC BY). The use, distribution or reproduction in other forums is permitted, provided the original author(s) or licensor are credited and that the original publication in this journal is cited, in accordance with accepted academic practice. No use, distribution or reproduction is permitted which does not comply with these terms. 